\title{
Relation between Perceived Stress, Depression, and Social Support with the Quality of Life in Women with Breast Cancer in Isfahan City
}

\author{
Rasoul Sabaei Dardashti ${ }^{1,}{ }^{*}$, Fatmeh Dehghan ${ }^{2}$, Arezou Hajiazizi ${ }^{3}$ \\ ${ }^{1}$ Department of Psychology, Faculty of Psychology and Educational Sciences, Islamic \\ Azad University, Khorasgan Branch, Isfahan, Iran \\ ${ }^{2}$ Department of Psychology, Razi University, Tehran, Iran \\ ${ }^{3}$ Department of Clinical Psychology, Islamic Azad University, Isfahan Branch, \\ Research Branch, Isfahan, Iran \\ ${ }^{*}$ Corresponding author: Rasoul Sabaei Dardashti, Department of Psychology, Faculty \\ of Psychology and Educational Sciences, Islamic Azad University, Khorasgan Branch, \\ Isfahan, Iran.E-mail: rasoul.sabaei@yahoo.com
}

DOI: $10.21859 /$ mci-supp- 84

Keywords:

Perceived Stress

Depression

Social Support

Quality of Life

Breast Cancer

\begin{abstract}
Introduction: Profound emotional problems and impairments such as stress, anxiety, and depression are contributed by cancer diagnosis for patients and their families. However, quality of life ( $\mathrm{QLL})$ is the most affected aspect among other ones that is associated by cancer. Accordingly, the following study examined the relation between perceived stress, depression, and social supports with the quality of life in women with breast cancer in Isfahan city.

Materials and Methods: The used method in this study is descriptive and correlational. The sample was 100 women with breast cancer in Isfahan city whom were selected voluntarily in 2017. For data collection, the Beck depression inventory, the social support from family and friends scale, and quality of life questionnaire (WHOQOL-BREF) were used. Results: The collected analyzed data by Pearson correlation coefficient showed that there is a notable negative correlation $(\mathrm{P}<0.001)$ between perceived stress and depression with quality of life in women with breast cancer. Simultaneous results of regression analysis also demonstrate perceived stress and social support can substantially predict the quality of life among women patients with breast cancer $(\mathrm{P}<0.001)$.

Conclusions: According to the results of this study, it can be claimed that social support of families is a determinant factor in quality of life of these patients.
\end{abstract}

